12,09

\title{
Спектроскопия плазмон-экситонов в наноструктурах полупроводник-металл
}

\author{
(C) В.А. Кособукин \\ Физико-технический институт им. А.Ф. Иофрфе РАН, \\ Санкт-Петербург, Россия \\ E-mail: Vladimir.Kosobukin@mail.ioffe.ru
}

\begin{abstract}
Представлены результаты теории смешанных плазмон-экситонных мод и их спектроскопии. Плазмон-экситоны образуются вследствие сильной кулоновской связи между квазидвумерными экситонами квантовой ямы и дипольными плазмонами наночастиц. Эффективная поляризуемость, связанная с наночастицей, вычислена в самосогласованном приближении с учетом локального поля дипольных плазмонов слоя и зарядов их изображения, обусловленных экситонной поляризацией близкой квантовой ямы. Исследованы спектры упругого рассеяния и зеркального отражения света в случаях отдельной наночастицы серебра и монослоя таких частиц соответственно, расположенных вблизи квантовой ямы GaAs/AlGaAs. Оптические спектры показывают наличие двухпиковой структуры с узким и глубоким провалом в области плазмон-экситонного резонанса. Обсуждается распространение плазмон-экситонных поляритонов в периодических сверхрешетках с элементарной ячейкой, образованной квантовой ямой и слоем металлических частиц. Исследован режим сверхизлучения, обусловленный брэгговской дифракцией плазмон-экситонных поляритонов на сверхрешетке. Показано, что широкий спектр плазмонного отражения, зависящий от числа ячеек в сверхрешетке, также имеет узкий и глубокий провал на частоте экситона.
\end{abstract}

DOI: $10.21883 /$ FTT.2018.08.46256.18Gr

\section{1. Введение}

Экситоны и плазмоны являются электронными коллективными возбуждениями, которые обусловлены дальнодействующим кулоновским взаимодействием носителей заряда. Экситонные состояния существуют в запрещенной энергетической зоне полупроводника, а плазменные колебания возникают в спектре электронного газа металлов. Оба типа возбуждений видимого диапазона могут сосуществовать в системах металл-полупроводник, важных для нанофотоники и приложений [1]. В связи с этим понимание особенностей плазмон-экситонного взаимодействия и особенностей его проявления в оптике является актуальной проблемой.

Сравнительно давно была поставлена задача о влиянии плазмонов на время жизни экситонов большого радиуса на границе раздела полупроводник-металл [2]. Для квантовой ямы теоретически изучались локализация экситонов и возрастание их силы осциллятора под влиянием близко расположенной металлической частицы [3]. Что касается экситонных структур с металлическими включениями, то в их спектрах уже наблюдались особенности, обусловленные резонансным взаимодействием между плазмонами и экситонами как малого, так и большого радиуса, ссылки имеются в [4-6]. Особо отметим наблюдение расщепления Раби большой величины для спектральной полосы, относящейся к связанным состояниям молекулярных экситонов $J$-агрегатов и локальных плазмонов благородных металлов [7-9]. Аналогичный эффект наблюдался для экситонов большого радиуса квантовой ямы на основе $\mathrm{ZnO}$, сильно связанных с плазмонами упорядоченного массива нанодисков Al [10]. Теоретически спектры плазмон-экситонов изучались в раз- ных моделях [4-6], включающих квантовые ямы с близко расположенными металлическими наночастицами.

В данной работе обсуждаются результаты теории сильной связи низкоразмерных экситонов большого радиуса с локальными плазмонами. Исследуется проявление связанных плазмон-экситонных возбуждений в резонансной спектроскопии упругого рассеяния света частицей и отражения света от массива частиц при сильном взаимодействии их плазмонов с экситонами близко расположенной квантовой ямы. Решается также задача о распространении электромагнитных волн (поляритонов) в сверхрешетках в спектральной области плазмон-экситонных резонансов.

\section{2. Модель экситонов и плазмонов}

Далее обсуждаются свойства плазмон-экситонов в трех принципиально разных моделях наноструктур полупроводник-металл [4-6], поддерживающих квазидвумерные экситоны и дипольные поверхностные (локальные) плазмоны. Общим для этих структур является наличие одной или многих квантовых ям в полупроводнике c фоновой проницаемостью $\varepsilon_{b}$. Около квантовой ямы расположена металлическая наночастица [4] или слой частиц [5], причем на основе последней модели рассматриваются периодические плазмон-экситонные сверхрешетки [6]. Ширина квантовых ям, размеры частиц, расстояния между частицами в слое и между слоем частиц и ближайшей квантовой ямой имеют нанометровый масштаб. Это обеспечивает кулоновский характер взаимодействия между плазмонами слоя наночастиц и квазидвумерными нерадиационными (темными) экситонами соседней квантовой ямы. Амплитуда поля куло- 
новских плазмон-экситонных мод затухает при удалении от элемента структуры „квантовая яма + слой наночастиц“. При наличии металлической наночастицы около квантовой ямы возникает плазмон-экситонный комплекс, приводящий к резонансному упругому рассеянию света. В случае упорядоченного короткопериодного слоя частиц вблизи ямы резонансные плазмон-экситонные особенности появляются в спектре зеркального отражения света. В периодической сверхрешетке, образованной плазмон-экситонными ячейками, возникают поляритоны, которые могут испытывать резонансную брэгговскую дифракцию в направлении оси сверхрешетки.

Будем считать, что в отсутствие наночастиц многослойная диэлектрическая среда с квантовыми ямами оптически однородна и изотропна в плоскости ям $x y$. Решения уравнений электродинамики для электрического поля и функций Грина имеют вид волн $F(z ; \kappa) \exp (i \kappa x-i \omega t)$ с частотой $\omega$, латеральным волновым вектором $\boldsymbol{\kappa}=\kappa \mathbf{e}_{x}$ и линейной поляризацией $p$ или $s$-типа. В случае последовательности квантовых ям, центрированных в плоскостях $z=z_{n, e}$ с номерами $n$, квазидвумерные экситоны ям дают вклад в поляризацию [11]

$$
P_{\alpha}^{\mathrm{I}}(z ; \kappa)=T_{0}(\omega) \sum_{n} \Psi\left(z-z_{n, e}\right) \int d z^{\prime} \Psi\left(z^{\prime}-z_{n, e}\right) E_{\alpha}\left(z^{\prime} ; \kappa\right)
$$

Резонансный член

$$
T_{0}(\omega)=\frac{\Gamma_{0}}{\omega_{0}-\omega-i \Gamma}
$$

включает частоту $\omega_{0}$ основного экситонного состояния, а также постоянные его нерадиационного Г и радиационного $\Gamma_{0}$ затухания. Огибающая волновой функции экситона $\Psi(z)=\Psi(-z)$ нормирована условием $\int d z \Psi^{2}(z)=1$, при этом $\int d z \Psi(z)=\sqrt{l}$, где $l-$ ширина квантовой ямы.

Вклад в поляризацию слоя эллипсоидальных частиц с центрами $\left(\boldsymbol{\rho}_{\mathbf{m}}, z_{n, p}\right)$ представим в виде [12]

$$
P_{\alpha}^{\mathrm{II}}(z, \boldsymbol{\rho})=\chi_{\alpha \alpha}^{(0)}(\omega) \sum_{n, \mathbf{m}} \delta\left(z-z_{n, p}\right) \delta\left(\boldsymbol{\rho}-\boldsymbol{\rho}_{\mathbf{m}}\right) E_{\alpha}\left(z_{n, p}, \boldsymbol{\rho}_{\mathbf{m}}\right) .
$$

Тензор поляризуемости металлической наночастицы, связанный с ее дипольным поверхностным плазмоном, имеет диагональные компоненты [12]

$$
\chi_{\alpha \alpha}^{(0)}(\omega)=\frac{a_{x} a_{y} a_{z}}{3} \frac{\Omega_{\alpha}^{2}}{\omega_{\alpha}^{2}-\omega^{2}-i \omega \gamma},
$$

которые соответствуют поляризации плазмона вдоль $\alpha$-й полуоси эллипсоида длиной $a_{\alpha}$. Выражение (4) получено для металла с проницаемостью $\varepsilon(\omega)=\varepsilon_{\infty}$ $-\omega_{p}^{2} /\left(\omega^{2}+i \omega \gamma\right)$, где $\omega_{p}-$ плазменная частота, $1 / \gamma$ - время релаксации электронов. В формуле (4)

$$
\left(\omega_{\alpha}^{(0)}\right)^{2}=\omega_{p}^{2} \frac{N^{(\alpha)}}{\mathcal{E}_{*}^{(\alpha)}}, \quad \Omega_{\alpha}^{2}=\omega_{\alpha}^{2} \frac{\varepsilon_{b}}{N^{(\alpha)} \varepsilon_{*}^{(\alpha)}},
$$

где $\varepsilon_{*}^{(\alpha)}=\left(\varepsilon_{\infty}-\varepsilon_{b}\right) N^{(\alpha)}+\varepsilon_{b}$. Частоты (5) зависят от длин $a_{x}, a_{y}, a_{z}$ полуосей эллипсоида через его коэффициенты деполяризации $N^{(\alpha)}[13]$.
Спектральные свойства квазидвумерных экситонов и локальных плазмонов с близкими частотами $\omega_{0} \approx \omega_{\alpha}$, входящими в (2) и (4), существенно различаются. Экситонные резонансы очень узкие $\left(\Gamma / \omega_{0} \ll 1\right)$, а плазмонные - весьма широкие $(\gamma / \Gamma \gg 1)$, но при этом добротность плазмонных резонансов имеет значительную величину $\omega_{\alpha} / \gamma \gg 1$.

Базовым элементом обсуждаемых далее моделей являются близко расположенные квантовая яма с квазидвумерными экситонами и слой металлических наночастиц, обладающих поверхностными плазмонами. Взаимодействие плазмонов в слое наночастиц приводит к образованию слоевых коллективных плазмонных мод (приближение сильной связи для локальных плазмонов [12]). В видимом диапазоне кулоновские моды плазмон-экситонов образуются при сильной связи мод: затухающих вдоль нормали слоевых плазмонов и нерадиационных (темных) экситонов ближайщей квантовой ямы. Далее мы проанализируем поляризуемость плазмон-экситонных возбуждений и связанные с ними наблюдаемые величины.

\section{3. Эффективная плазмон-экситонная поляризуемость}

Оптические явления с участием плазмон-экситонов анализируются в рамках теории многократного резонансного рассеяния на дипольных комплексах. Качественно такой комплекс представляется точечным диполем с эффективной поляризуемостью $\hat{\chi}$, обусловленной откликом частицы и зарядом изображения вследствие поляризации квантовой ямы. Поле излучения, обусловленное слоем эффективных диполей с поляризуемостью $\hat{\chi}$, центрированных в точках $\left(\boldsymbol{\rho}_{\mathbf{m}}, z_{n}\right)$, выражается формулой $[6,12]$

$$
\begin{aligned}
E_{\beta}(\mathbf{r})-\delta_{\alpha \beta} E_{\alpha}^{(0)}(\mathbf{r})= & \sum_{n, \mathbf{m}} D_{\beta \alpha}^{(0)}\left(z, z_{n}, \boldsymbol{\rho}-\boldsymbol{\rho}_{\mathbf{m}}\right) \chi_{\alpha \alpha}(\omega) \\
& \times E_{\alpha}^{(0)}\left(z_{n}, \boldsymbol{\rho}_{\mathbf{m}}\right) .
\end{aligned}
$$

Здесь $\mathbf{E}^{(0)}(z, \boldsymbol{\rho})$ - поле и $\hat{D}^{(0)}\left(z, z^{\prime}, \boldsymbol{\rho}-\boldsymbol{\rho}^{\prime}\right)$ - тензорная функция Грина диэлектрической среды с квантовыми ямами в отсутствие частиц, которая трансляционно инвариантна в латеральных направлениях. Согласно (6), эффективная плазмон-экситонная поляризуемость $\hat{\chi}(\omega)$ является откликом наноструктуры на поле $\mathbf{E}^{(0)}=\mathbf{e}_{\alpha} E_{\alpha}^{(0)}$, не возмущенное плазмонами. В формуле (6) компоненты $\chi_{\alpha \alpha}(\omega)$ тензора эффективной поляризуемости, определяемые кулоновскими модами плазмон-экситонов, равны [4-6]

$$
\chi_{\alpha \alpha}(\omega)=\left[1 / \chi_{\alpha \alpha}^{(0)}(\omega)-\sigma^{(\alpha)}(\omega)\right]^{-1} .
$$

В это выражение входит компонента $\chi_{\alpha \alpha}^{(0)}$ плазмонной поляризуемости частицы, определяемая формулами (4), (5). Величины $\sigma^{(\alpha)}(\omega)$ учитывают резонансный 

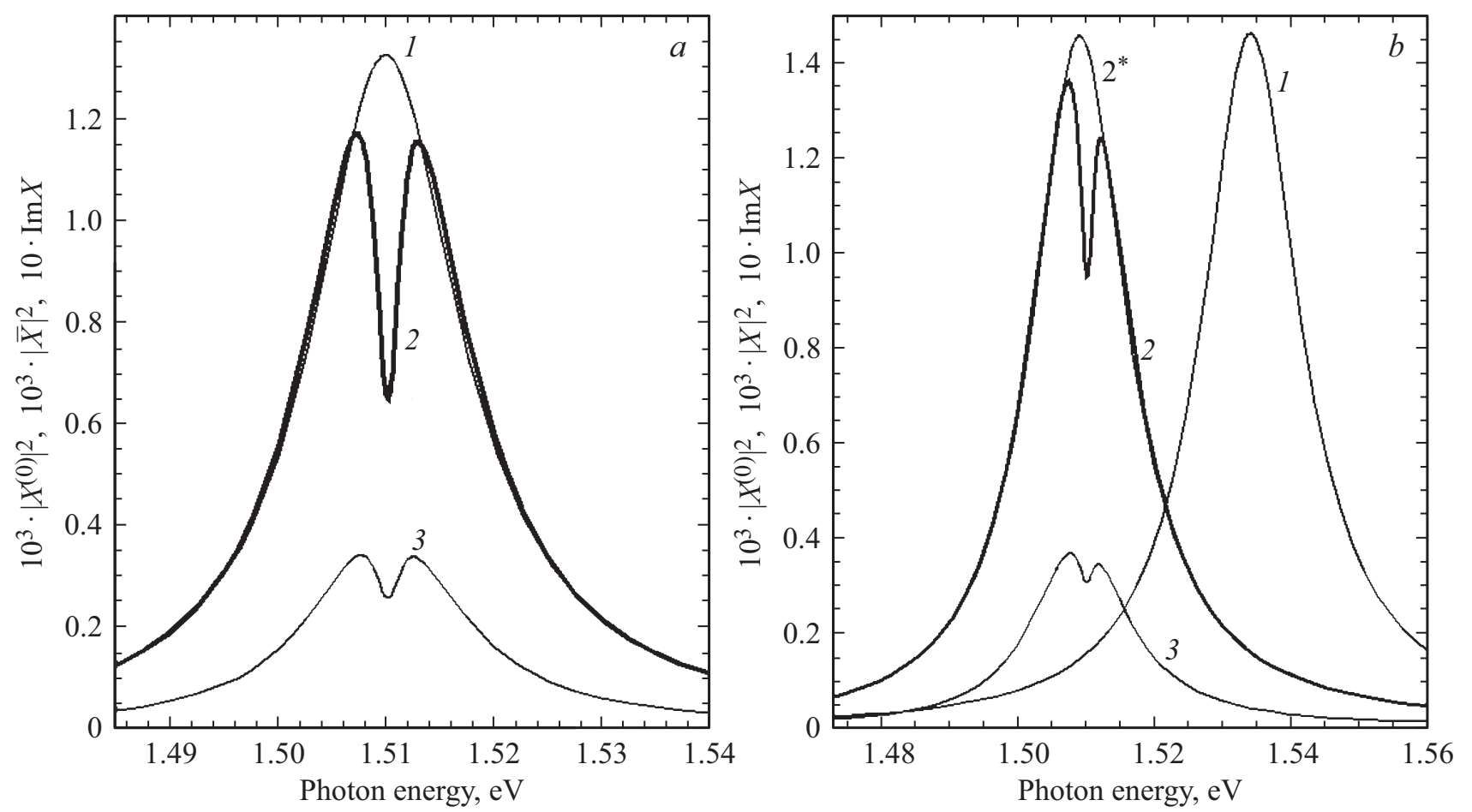

Рис. 1. Спектры плазмон-экситонной поляризуемости $\left|X^{(0)}\right|^{2}=k_{0}^{6}\left|\chi_{y y}^{(0)}\right|^{2},|X|^{2}=k_{0}^{6}\left|\chi_{y y}\right|^{2}$ и $\operatorname{Im} X=k_{0}^{3} \operatorname{Im} \chi_{y y}$ в случаях одного нанодиска $\mathrm{Ag}(a)$ и слоя нанодисков $\mathrm{Ag}(b)$, находящихся вблизи квантовой ямы $\mathrm{AlGaAs} / \mathrm{GaAs} . a-$ спектры $\left|X^{(0)}\right|^{2}(1),|\bar{X}|^{2}(2)$ и $\operatorname{Im} X(3)$ при $\eta=a_{z} / a_{x}=0.586, h=10.5 \mathrm{~nm} . b-$ спектры $\left|X^{(0)}\right|^{2}(1),|X|^{2}$ с учетом сумм $S_{d}^{(\alpha)}\left(2^{*}\right),|X|^{2}(2)$ и $\operatorname{Im} X(3)$ с учетом сумм $S_{d}^{(\alpha)}+S_{i}^{(\alpha)}$ при $\eta=0.615, h=9 \mathrm{~nm}, A=22 \mathrm{~nm}$. Принято $\hbar \omega_{0}=1.51 \mathrm{eV}, \hbar \Gamma_{0}=0.25 \mathrm{eV}, \hbar \Gamma=1 \mathrm{meV}, \varepsilon_{b}=12, l=8 \mathrm{~nm}$ для квантовой ямы $\mathrm{AlGaAs} / \mathrm{GaAs}$ и $a_{x}=10 \mathrm{~nm}$ для нанодисков с проницаемостью $\mathrm{Ag}$ из [14].

эффект локального поля, т.е. действие на выделенный плазмон других плазмонов слоя и зарядов их изображения, обусловленных экситонной поляризацией квантовой ямы. Как следствие, тензор эффективной поляризуемости $\hat{\chi}$ с компонентами (7) описывает симметрию и резонансные свойства плазмон-экситонного комплекса, сформированного наночастицей и квантовой ямой.

Далее рассматриваем наночастицы в форме сфероидов (нанодисков) с $a_{x}=a_{y} \neq a_{z}, \chi_{x x}^{(0)}=\chi_{y y}^{(0)}$. Тогда для диполя изображения плазмона отдельной частицы, обусловленного экситонной поляризацией квантовой ямы, в выражении (7) стоит

$$
\bar{\sigma}^{(\alpha)}=\frac{3 \pi}{8} \frac{1}{\varepsilon_{b}^{2}} \frac{1}{h^{4}} F_{Q W}(\omega)
$$

в качестве $\sigma^{(\alpha)}$ с $\alpha=x, y$ [4]. Здесь спектральная функция

$$
F_{Q W}(\omega)=\frac{\Gamma_{0}}{\omega_{0}-\omega-i \Gamma}+\frac{\Gamma_{0}}{\omega_{0}+\left(4 \pi / \varepsilon_{b}\right) \Gamma_{0}-\omega-i \Gamma}
$$

связана с нерадиационными (темными) экситонами квантовой ямы, поляризованными по осям $x$ и $z$. Малая величина $\left(4 \pi / \varepsilon_{b}\right) \Gamma_{0} \ll \Gamma$ определяет анизотропию их частот.
Оценим вклад в (7) для модели точечных плазмонных диполей в слое одинаковых сфероидов с центрами в узлах

$$
\boldsymbol{\rho}_{\mathbf{m}}=A \cdot \mathbf{m}, \quad \mathbf{m}=\mathbf{e}_{x} \cdot m_{1}+\mathbf{e}_{y} \cdot m_{2}
$$

квадратной решетки с периодом $A$. Решетка расположена на расстоянии $h \ll\left(k_{0} \sqrt{\varepsilon_{b}}\right)^{-1}$ от квантовой ямы, где $k_{0}=\omega / c, c-$ скорость света. В квазистатическом приближении [5]

$$
\sigma^{(\alpha)}(\omega)=\frac{1}{\varepsilon_{b} A^{3}}\left(S_{d}^{(\alpha)}+\frac{12 \pi h l}{\varepsilon_{b} A^{2}} F_{Q W}(\omega) S_{i}^{(\alpha)}\right) .
$$

Вклад (11) в эффективную поляризуемость (7), обусловленный экситонами, включает безразмерные суммы

$$
S_{d}^{(\alpha)}=A^{3} \sum_{\mathbf{m}(\neq 0)} \frac{3 \rho_{\mathbf{m}, \alpha}^{2}-\rho_{\mathbf{m}}^{2}}{\rho_{\mathbf{m}}^{5}},
$$

$$
S_{i}^{(\alpha)}(h)=\left(\frac{A}{2 h}\right)^{5}-A^{5} \sum_{\mathbf{m}(\neq 0)} \frac{5 \rho_{\mathbf{m}, \alpha}^{2}-\rho_{\mathbf{m}}^{2}-4 h^{2}}{\left(\rho_{\mathbf{m}}^{2}+4 h^{2}\right)^{7 / 2}}
$$

по узлам m решетки (10), которые выражают локальное (действующее) поле на заданном узле решетки (частице массива). Сумма $S_{d}^{(\alpha)}$ определяет поле, обусловленное дипольными плазмонами других частиц слоя (10), а сумма $S_{i}^{(\alpha)}$ учитывает поле зарядов их изображения, обусловленных наличием квантовой ямы. 
Частота плазмонов $\omega_{\alpha}$, зависящая от отношения полуосей сфероидов $\eta=a_{z} / a_{x}$, их размера и периода решетки, варьируется при фиксированной частоте экситона $\omega_{0}$. Когда частоты плазмонов и экситонов существенно различаются $\left(\left|\omega_{\alpha}-\omega_{0}\right| \gg \Gamma\right)$, слабый экситонный пик поляризуемости проявляется независимо на фоне сильного плазмонного пика [4]. При резонансе $\omega_{\alpha}=\omega_{0}$ спектры плазмон-экситонной поляризуемости показаны кривыми 2 на рис. $1, a$ и $b$. Эти спектры вычислены по формуле (7) с функцией (8) в случае одной наночастицы и с функцией (11), (12) в случае слоя взаимодействующих частиц. Результаты относятся к нанодискам $\mathrm{Ag}$ с $a_{x}=a_{y} \neq a_{z}$, расположенным вблизи квантовой ямы $\mathrm{AlGaAs} / \mathrm{GaAs}$. При обсуждении исходным считаем спектр поляризуемости $\left|\chi_{\alpha \alpha}^{(0)}(\omega)\right|^{2}$ нанодисков, находящихся в однородной среде с проницаемостью $\varepsilon_{b}$ (кривые 1 на рис. $1, a$ и $b$ ).

Для дипольных плазмонов отдельной частицы учет зарядов изображения, обусловленных экситонной поляризацией квантовой ямы, приводит к плазмон-экситонному расщеплению спектра поляризуемости $\left|\bar{\chi}_{y y}(\omega)\right|^{2}$, которое при резонансе $\bar{\omega}_{\alpha}=\omega_{0}$ демонстрируется кривой 2 на рис. $1, a$. Наличие плазмон-экситонного расщепления спектра подтверждается двухпиковой структурой величины $\operatorname{Im} \bar{\chi}_{y y}(\omega)$ с $\bar{\chi}_{\alpha \alpha}=\chi_{\alpha \alpha}^{(0)} /\left(1-\chi_{\alpha \alpha}^{(0)} \bar{\sigma}^{(\alpha)}\right)$ (кривая 3 на рис. $1, a)$, которая определяет спектральную зависимость поглощения света.

На рис. 1, $b$ представлена поляризуемость плазмонэкситонного комплекса в случае слоя наночастиц вблизи квантовой ямы. Показан последовательный переход от спектра 1 поляризуемости $\left|\chi_{x x}^{(0)}(\omega)\right|^{2}$ изолированной частицы к спектру $2^{*}$, учитывающему только сумму $S_{d}^{(\alpha)}$ из (12) для внутрислоевого взаимодействия дипольных плазмонов, а затем к спектру 2, учитывающему также сумму $S_{i}^{(\alpha)}$ из (12), связанную с зарядами изображения. Наличие плазмон-экситонного расщепления демонстрирует величина $\operatorname{Im} \chi_{\alpha \alpha}(\omega)$ (кривая 3 на рис. $\left.1, b\right)$, определяющая спектр оптического поглощения.

Таким образом, при резонансе $\omega_{\alpha}=\omega_{0}$ спектр плазмон-экситонной поляризуемости состоит из двух пиков, разделенных узким провалом на частоте резонанса. Эти пики указывают на наличие расщепления Раби, что подтверждается двухпиковой структурой величин $\operatorname{Im} \chi_{\alpha \alpha}(\omega)$ на рис. $1, a$ и $b$, которые характеризуют спектральную зависимость поглощения света при наличии связи между плазмонами и экситонами.

\section{4. Спектроскопия плазмон-экситонов}

Обсудим приложения теории для расчета величин, наблюдаемых основными методами спектроскопии.

4.1. Упругое рассеяние света. В случае наночастицы, находящейся около квантовой ямы, возникает упругое рассеяние света. Наблюдаемой величиной является дифференциальное сечение рассеяния $d \sigma_{\lambda \rightarrow \lambda^{\prime}} / d \Omega^{\prime}=r^{2}\left|\mathbf{S}_{\lambda^{\prime}}\right| /\left|\mathbf{S}_{\lambda}\right|$, где $\mathbf{S}_{\lambda}-$ вектор Пойнтинга, в элемент телесного угла $d \Omega^{\prime}=\sin \theta^{\prime} d \theta^{\prime} d \varphi^{\prime}$ с преобразованием линейной поляризации света $\lambda \rightarrow \lambda^{\prime}$. Следуя [4], получаем сечение

$$
\begin{aligned}
& \frac{d \sigma_{s \rightarrow \lambda^{\prime}}}{d \Omega^{\prime}}=k_{0}^{4}\left|\bar{\chi}_{y y}(\omega)\right|^{2} \\
& \quad \times\left|1+\rho_{Q W}^{\lambda^{\prime}}\left(\kappa^{\prime}\right) e^{2 i k_{b}\left(\kappa^{\prime}\right) h}\right|^{2} \cdot\left|1+\rho_{Q W}^{s}(\kappa) e^{2 i k_{b}(\kappa) h}\right|^{2} F_{\lambda^{\prime}}\left(\Omega^{\prime}\right)
\end{aligned}
$$

плазмон-экситонного рассеяния $s$-поляризованного света в каналах $s \rightarrow \lambda^{\prime}$, где $\lambda^{\prime}=s, p$. Здесь $\rho_{Q W}^{\lambda}(\kappa)-$ коэффициент отражения от квантовой ямы световой волны $\mathrm{c}$ поляризацией $\lambda$ и проекцией волнового вектора $\kappa$ на плоскость ямы. Резонансная структура спектра (13) определяется компонентами $\left|\bar{\chi}_{y y}\right|^{2}=\left|\chi_{y y}^{(0)} /\left(1-\chi_{y y}^{(0)} \bar{\sigma}^{(y)}\right)\right|^{2}$ эффективной поляризуемости плазмон-экситонного комплекса с $\bar{\sigma}^{(y)}$ из (8). Диаграммы направленности излучения $F_{s}\left(\Omega^{\prime}\right)=\cos ^{2} \varphi^{\prime}$ и $F_{p}\left(\Omega^{\prime}\right)=\cos ^{2} \theta^{\prime} \sin ^{2} \varphi^{\prime}$, выраженные через углы рассеяния $\theta^{\prime}$ и $\varphi^{\prime}$, показывают, что при $\varphi^{\prime}=0$ и $\varphi^{\prime}=\pi$ возможно только рассеяние $s \rightarrow s$, а при других азимутальных углах $\varphi^{\prime}$ наряду с $s \rightarrow s$ появляется рассеяние $s \rightarrow p$. Наличие когерентных волн с поляризациями $s$ и $p$ означает, что при $\varphi^{\prime} \neq 0, \pi$ поле в заданном направлении рассеяния имеет эллиптическую поляризацию, резонансно зависящую от частот плазмон-экситонов. В рамках представленной здесь теории можно также исследовать рассеяние света в круговые плазмон-экситоны с $\kappa^{\prime} \gg k_{0} \sqrt{\varepsilon_{b}}$ по аналогии с возбуждением точечным

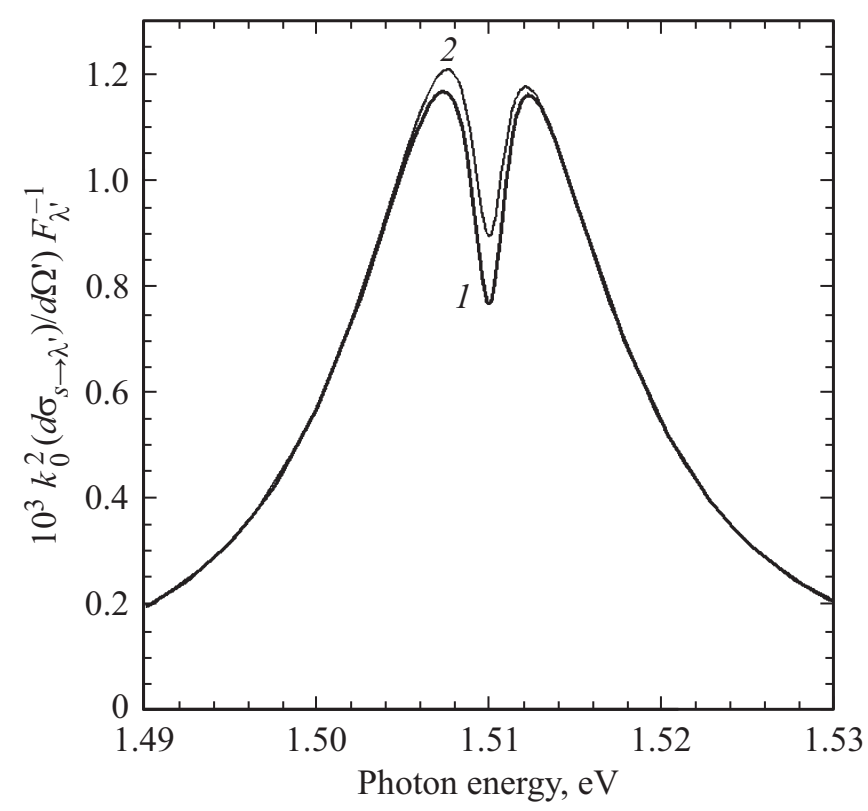

Рис. 2. Спектральные зависимости $k_{0}^{2}\left(d \sigma_{s \rightarrow \lambda^{\prime}} / d \Omega^{\prime}\right) F_{\lambda^{\prime}}^{-1}$ сечений рассеяния $s$-поляризованного света в волны с поляризациями $\lambda^{\prime}=s(1)$ и $\lambda^{\prime}=p(2)$ нанодиском $\mathrm{Ag}$, находящимся вблизи квантовой ямы $\mathrm{AlGaAs} / \mathrm{GaAs}$. Вычислено по формулам (7) -(9) и (13) при $\theta=0$ и $\theta^{\prime}=60^{\circ}$ с $a_{x}=10 \mathrm{~nm}, \eta=0.585$, $l=8 \mathrm{~nm}$ и $h=10.5 \mathrm{~nm}$ и теми же экситонными параметрами, что на рис. 1. 
диполем нерадиационных плазмонов на плоской поверхности металла [15].

На рис. 2 показаны спектры рассеяния $d \sigma_{s \rightarrow \lambda^{\prime}} / d \Omega^{\prime}$ при резонансе $\bar{\omega}_{y}=\omega_{0}$, вычисленные по формулам (7)-(9) и (13). Видно, что сечения рассеяния имеют те же спектральные особенности, что и спектр плазмон-экситонной поляризуемости (кривая 2 на рис. 1,a). Сечения рассеяния (13) в каналах $s \rightarrow s \quad$ и $s \rightarrow p$ слабо отличаются друг от друга из-за малости коэффициента экситонного отражения квантовой ямы $\left|\rho_{Q W}^{\lambda}\right| \sim k_{0} l \Gamma_{0} /\left(\Gamma_{0}+\Gamma\right) \ll 1$.

4.2. Отражение света. Для слоя металлических наночастиц, расположенных вблизи квантовой ямы, используется модель квадратной решетки (10) с периодом $A$. Вообще говоря, при возбуждении плазмон-экситонов на решетке должна возникать резонансная брэгговская дифракция, определяемая латеральными векторами обратной решетки $|\mathbf{b}| \sim 2 \pi / A$. Рассматриваемые короткопериодные (плотные) решетки с $A \ll 1 /\left(k_{0} \sqrt{\varepsilon_{b}}\right)$ обеспечивают дополнительный вклад только в зеркальное отражение света. В результате для коэффициента отражения $s$-поляризованной волны находим [5]

$$
R=\left|\rho_{Q W}^{s}+\frac{2 \pi i k_{0}^{2}}{k_{b} A^{2}} \chi_{y y}\left(e^{-i k_{b} h}+\rho_{Q W}^{s} e^{i k_{b} h}\right)^{2}\right|^{2},
$$

где $\chi_{y y}=\chi_{y y}^{(0)} /\left(1-\chi_{y y}^{(0)} \sigma^{(y)}\right)$ включает $\sigma^{(y)}$ из (11). На рис. 3 коэффициент зеркального плазмон-экситонного

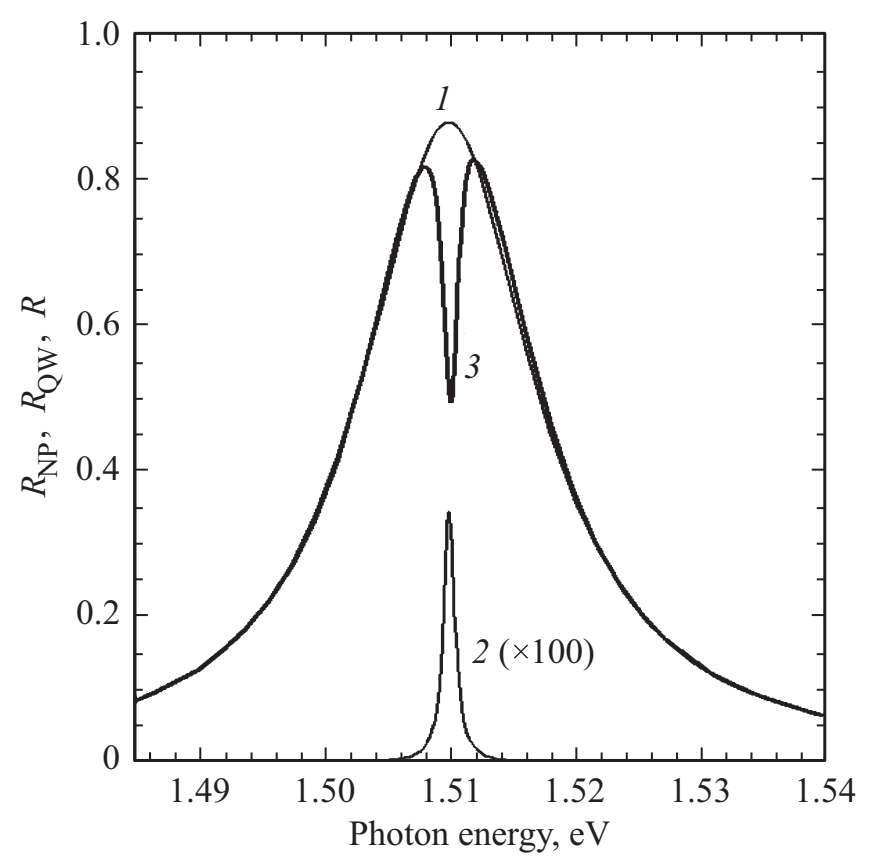

Рис. 3. Коэффициенты нормального отражения света от монослоя серебряных нанодисков $R_{N P}(1)$, от квантовой ямы $\mathrm{AlGaAs} / \mathrm{GaAs} R_{Q W}(2)$ (умножено на 100$)$ и от близко расположенных слоя нанодисков и квантовой ямы $R(3)$. Вычислено по формуле (14) при $\omega_{\alpha}=\omega_{0}, \hbar \Gamma=0.5 \mathrm{meV}, A=25 \mathrm{~nm}$, $a_{x}=a_{y}=8 \mathrm{~nm}, \eta=a_{z} / a_{x}=0.596, l=9 \mathrm{~nm}, h=9 \mathrm{~nm}$ и с теми же другими экситонными параметрами, что на рис. 1. отражения $s$-поляризованного света (14) при нормальном падении показан в сравнении с коэффициентами экситонного $R_{Q W}=\left|\rho_{Q W}^{s}\right|^{2}$ и плазмонного $R_{N P}$ (при $\left.\rho_{Q W}^{s}=0\right)$ отражения. Видно, что при $\omega_{\alpha}=\omega_{0}$ плазмонэкситонное взаимодействие приводит к двухпиковой структуре спектра со значительной экситонной особенностью в виде провала. Глубина провала в несколько раз превышает масштаб экситонного пика отражения $R_{Q W}$, и это позволяет говорить об усилении экситонной спектральной особенности плазмонами. Однако следует отметить, что в приближении (6) для вторичных волн эффект отражения света от слоя наночастиц может быть несколько завышен вследствие условия $\Omega_{\alpha} \gg \gamma$ для плазмонов в выражениях (4) и (7).

4.3. Плазмон-экситонные поляритоны в с ве рх реше тках. В области плазмон-экситонных резонансов электромагнитные волны распространяются в форме поляритонов. Теория плазмон-экситонных поляритонов рассматривалась для сверхрешеток, т.е. периодических последовательностей элементарных сверхячеек, образованных близко расположенными квантовой ямой и слоем металлических наночастиц [6]. Как указано выше, внутри ячеек с $h \ll 1 /\left(k_{0} \sqrt{\varepsilon_{b}}\right)$ взаимодействие экситонов и плазмонов осуществляется через их кулоновское (ближнее) поле. Взаимодействие между плазмон-экситонными возбуждениями разных ячеек реализуется через запаздывающее электромагнитное поле, которое имеет интерференционный характер. Самосогласованное решение задачи о распространении плазмон-экситонных поляритонов получается методом матриц переноса [6]. При нормальном распространении поляритонов матрицы переноса выражаются через коэффициент плазмон-экситонного отражения света элементарной ячейкой сверхрешетки

$$
\rho=\rho_{Q W}+\rho_{N P}\left(1+\rho_{Q W}\right)^{2} .
$$

Эта формула включает экситонный коэффициент отражения квантовой ямой $\rho_{Q W}$ и плазмонный коэффициент отражения $\rho_{N P}$ слоем наночастиц. Плазмон-экситонные поляритоны блоховского типа, распространяющиеся вдоль оси сверхрешетки, имеют одномерный закон дисперсии в зависимости от волнового числа $q(\omega)$.

Особый интерес связан с брэгговскими сверхрешетками, период которых $d$ удовлетворяет условию Брэгга $q\left(\omega_{0}\right)=\pi / d$ при частоте резонанса $\omega_{\alpha}=\omega_{0}$. Коэффициент плазмон-экситонного отражения света брэгговской сверхрешеткой с числом $N$ элементарных ячеек равен [6]

$$
R_{N}=\frac{N^{2}|\rho|^{2}}{|N \rho-\tau|^{2}}
$$

Из формулы (16) с $\tau=1+\rho$ следует наличие следующих режимов распространения поляритонов: 1) сверхизлучение при $N|\rho| \ll 1$, когда коэффициент отражения $R_{N} \approx N^{2}|\rho|^{2}$ и ширина спектра возрастают с числом элементарных ячеек $N$, и 2) фотонно- 


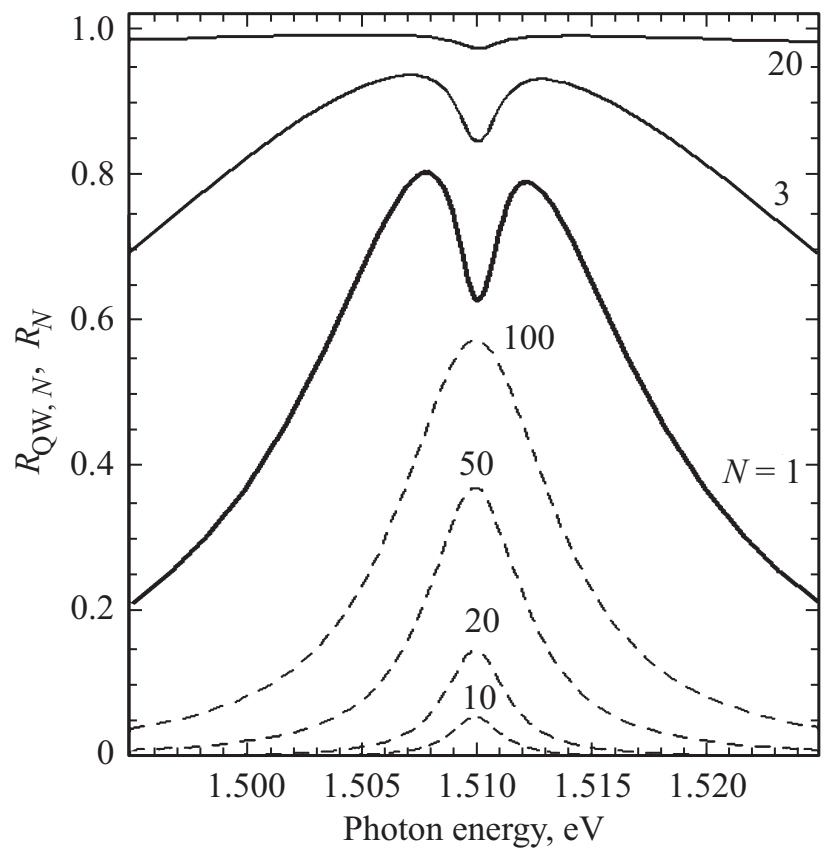

Pис. 4. Спектры нормального экситонного $R_{Q W, N}$ (штриховые линии) и плазмон-экситонного $R_{N}$ (сплошные линии) отражения света в режиме сверхизлучения для периодических сверхрешеток с $N$ ячейками, состоящими из слоя нанодисков $\mathrm{Ag}$ и квантовой ямы $\mathrm{GaAs} / \mathrm{AlGaAs}$. Спектры обозначаются цифрой, которая показывает число $N$ ячеек в сверхрешетке. Вычислено по формулам (15), (16) при резонансе $\omega_{\alpha}=\omega_{0}$, $A=25 \mathrm{~nm}, a_{x}=a_{y}=8 \mathrm{~nm}, \eta=0.596, l=9 \mathrm{~nm}, h=9 \mathrm{~nm} \mathrm{c}$ теми же экситонными параметрами, что на рис. 1.

кристаллический режим, который предполагает переход к полному отражению $R_{N} \rightarrow 1$ при больших числах $N$. Переход между этими двумя режимами в брэгговских сверхрешетках ранее изучался для экситонных поляритонов [16]. На рис. 4 представлены результаты расчета по формулам (15), (16) плазмон-экситонного коэффициента отражения $R_{N}(\omega)$ в зависимости от числа $N$ элементарных ячеек брэгговской структуры. Для сравнения штриховыми линиями показаны коэффициенты $R_{Q W . N}$ экситонного (при $\left.\rho_{N P}=0\right)$ отражения света в режиме сверхизлучения. Видно, что типичная для сверхизлучения зависимость спектра $R_{N}$ от $N$ проявля-

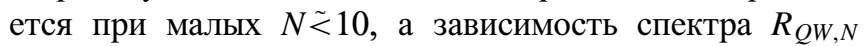
от $N$ в экситонных сверхрешетках проявляется до значений $N \sim 10^{3} \quad[6,16]$. Подавление сверхизлучения в сверхрешетке с плазмонами связано с относительно большой скоростью распада плазмонов по сравнению с экситонами. Из рис. 4 видно, что в спектре поляритонов плазмон-экситонное расщепление Раби также проявляется в форме двухпикового спектра с провалом на резонансной частоте экситона. Этот спектр как целое и особенности провала меняются при увеличении числа ячеек $N$ сверхрешетки, причем связанная с экситонами спектральная структура исчезает при сравнительно малых числах элементарных ячеек.

\section{5. Заключение}

Сильное резонансное кулоновское взаимодействие между локальными плазмонами слоя наночастиц и экситонами квантовой ямы приводит к образованию плазмон-экситонных мод и характерному расщеплению оптических спектров в области резонанса. Два спектральных пика, разделенных узким провалом, возникают в поляризуемости локализованных плазмон-экситонных комплексов. Это расщепление проявляется в плазмон-экситонных спектрах упругого рассеяния и зеркального отражения света. Провал в этих спектрах на частоте экситона можно интерпретировать в терминах усиления экситонного отклика плазмонами. Для сверхрешеток при малом числе плазмон-экситонных сверхячеек провал в спектре отражения проявляется в сверхизлучательном режиме, однако он исчезает при переходе к фотоннокристаллическому режиму при сравнительно небольшом увеличении числа сверхячеек. Представляется перспективным рассмотрение плазмон-экситонных эффектов в пропускании и поглощении света, а также в фотолюминесценции.

\section{Список литературы}

[1] A.A. Toropov, T.V. Shubina. Plasmonic Effects in MetalSemiconductor Structures. Univ. Press, Oxford (2015). $361 \mathrm{p}$.

[2] В.М. Агранович, М.Д. Галанин. Перенос энергии электронного возбуждения в конденсированных средах. Наука, M. (1978). 383 c.

[3] V.I. Sugakov, G.V. Vertsimakha. Phys. Rev. B 81, 235308 (2010).

[4] В.А. Кособукин. ФТТ 57, 1413 (2015).

[5] V.A. Kosobukin. Solid State Commun. 228, 43 (2016).

[6] В.А. Кособукин. ФТТ 59, 972 (2017).

[7] Y. Sugawara, T.A. Kelf, J.J. Baumberg, M.E. Abdelsalam, P.N. Bartlett. Phys. Rev. Lett. 97, 266808 (2006).

[8] S. Balci. Opt. Lett. 38, 4498 (2013).

[9] B.G. DeLacy, O.D. Miller, C.W. Hsu, Z. Zander, S. Lacey, R. Yagloski, A.W. Fountain, E. Valdes, E. Anquillare, M. Soljacic, S.G. Johnson, J.D. Joannopoulos. Nano Lett. 15, 2588 (2015).

[10] B.J. Lawrie, K.-W. Kim, D.P. Norton, R.F. Haglund, Jr. Nano Lett. 12, 6152 (2012).

[11] E.L. Ivchenko. Optical spectroscopy of semiconductor nanostructures. Alpha Science International, Ltd. (2005). 315 p.

[12] В.А. Кособукин, А.В. Коротченков. ФТТ 58, 2446 (2016).

[13] Л.Д. Ландау, Е.М. Лифшиц. Электродинамика сплошных сред. Наука, М. (1982). 620 с.

[14] P.B. Johnson, R.W. Christy. Phys. Rev. B 6, 4370 (1972).

[15] В.А. Кособукин. ФТТ 35, 884 (1993).

[16] Е.Л. Ивченко, А.Н. Поддубный. ФТТ 55, 833 (2013).

Редактор Ю.Э. Китаев 